\title{
The role of Stewartson and Ekman layers in turbulent rotating Rayleigh-Bénard convection
}

\author{
Rudie P.J. Kunnen ${ }^{1}$, Richard J.A.M. Stevens ${ }^{2}$, Jim Overkamp ${ }^{1}$, Chao \\ Sun $^{2}$, GertJan F. van Heijst ${ }^{1}$, Herman J.H. Clercx ${ }^{1,3}$ \\ ${ }^{1}$ Department of Physics and J.M. Burgers Centre for Fluid Dynamics, Eindhoven University of \\ Technology, P.O. Box 513, 5600 MB Eindhoven, The Netherlands \\ ${ }^{2}$ Department of Science and Technology and J.M. Burgers Centre for Fluid Dynamics, \\ University of Twente, P.O. Box 217, 7500 AE Enschede, The Netherlands, \\ ${ }^{3}$ Department of Applied Mathematics, University of Twente, P.O. Box 217, 7500 AE \\ Enschede, The Netherlands
}

\begin{abstract}
When the classical Rayleigh-Bénard (RB) system is rotated about its vertical axis roughly three regimes can be identified. In regime I (weak rotation) the large scale circulation (LSC) is the dominant feature of the flow. In regime II (moderate rotation) the LSC is replaced by vertically aligned vortices. Regime III (strong rotation) is characterized by suppression of the vertical velocity fluctuations. Using results from experiments and direct numerical simulations of RB convection for a cell with a diameter-to-height aspect ratio equal to one at $R a \sim 10^{8}-10^{9}(\operatorname{Pr}=4-6)$ and $0 \lesssim 1 / R o \lesssim 25$ we identified the characteristics of the azimuthal temperature profiles at the sidewall in the different regimes. In regime I the azimuthal wall temperature profile shows a cosine shape and a vertical temperature gradient due to plumes that travel with the LSC close to the sidewall. In regime II and III this cosine profile disappears, but the vertical wall temperature gradient is still observed. It turns out that the vertical wall temperature gradient in regimes II and III has a different origin than that observed in regime I. It is caused by boundary layer dynamics characteristic for rotating flows, which drives a secondary flow that transports hot fluid up the sidewall in the lower part of the container and cold fluid downwards along the sidewall in the top part.
\end{abstract}

\section{Introduction}

The classical system to study buoyancy driven flows is the Rayleigh-Bénard (RB) system (Ahlers et al. 2009, Lohse \& Xia 2010). In this system a layer of fluid is confined between two horizontal plates and is heated from below and cooled from above. The RB system is also very suitable to study the influence of rotation on heat transport mechanisms. In order to do this the RB system is rotated at an angular speed $\Omega$ about its vertical axis. Studies about the influence of rotation on heat transport are relevant to understand many geophysical and astrophysical flow phenomena such as the global thermohaline circulation, convection in the interior of gaseous planets, and convection in the outer layer of the Sun. Furthermore, the studies are also very relevant for optimization of industrial applications. Therefore, turbulent rotating convection has been studied extensively in laboratory experiments (for example, Rossby 1969 ; Boubnov \& Golitsyn 1986 . Fernando et al. 1991; Zhong et al. 1993, Liu \& Ecke 1997, Sakai 1997; Hart \& Olsen 1999, Vorobieff \& Ecke 2002; Kunnen et al.|2008b; Liu \& Ecke 2009; Niemela et al. 2010: Zhong 


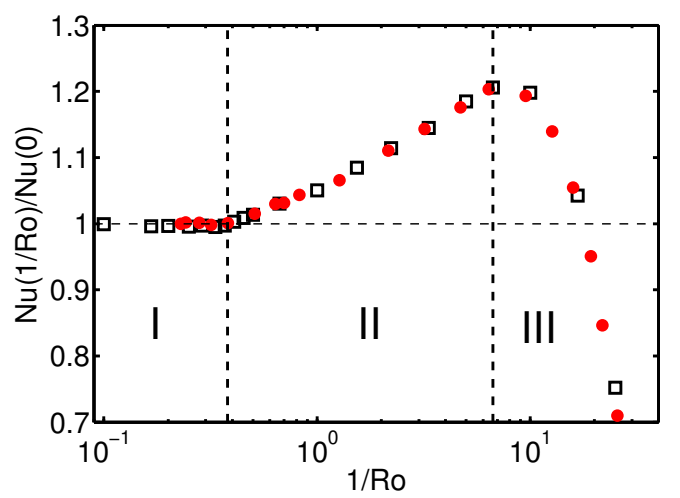

Figure 1. The scaled heat transfer $N u(1 / R o) / N u(0)$, with $N u$ the Nusselt number defined in Eq. 1.2), as function of $1 / R o$ on a logarithmic scale. Experimental and numerical data for $R a=2.73 \times 10^{8}$ and $\operatorname{Pr}=6.26$ are indicated by red dots and open squares, respectively. Data taken from Zhong et al. (2009) and Stevens et al. (2009) and are called run E2 in Zhong \& Ahlers (2010). The transition between the different regimes, see text, is indicated by the vertical dashed lines.

\& Ahlers 2010), direct numerical simulations (for example, Julien et al. $1996 b \mid a$; Kunnen et al. 2006: Schmitz \& Tilgner 2009, Kunnen et al. 2009, Schmitz \& Tilgner 2010; Stevens et al. $2010 c$ a), and with combined experimental and numerical investigations (Kunnen et al. 2008a; Zhong et al. 2009; Stevens et al. 2009; Kunnen et al. 2010a b; Weiss et al. 2010).

In this paper we indicate the rotation rate around the vertical axis by the Rossby number Ro, which compares the inertial and Coriolis forces in the system. Here, Ro is defined as

$$
R o=\sqrt{\frac{R a}{\operatorname{Pr} T a}}=\frac{1}{2 \Omega} \sqrt{\frac{\beta g \Delta}{L}},
$$

where $\operatorname{Pr}=\nu / \kappa$ is the Prandtl number, $T a=\left(2 \Omega L^{2} / \nu\right)^{2}$ is the Taylor number and $R a=\beta g L^{3} \Delta /(\nu \kappa)$ is the Rayleigh number, with $\Omega$ the rotation rate, $\beta$ the thermal expansion coefficient, $g$ the gravitational acceleration, $\nu$ the kinematic viscosity, $\kappa$ the thermal diffusion coefficient, and $\Delta$ the temperature difference between the two plates. Throughout the paper we will consider a cell with aspect ratio $\Gamma \equiv D / L=1$, with $L$ the height of the RB cell and $D$ its diameter. The dimensionless heat transport in the system is indicated by the Nusselt number,

$$
N u=\frac{Q L}{\lambda \Delta},
$$

where $Q$ is the heat-current density and $\lambda$ the thermal conductivity of the fluid in the absence of convection.

In figure 1 we show a typical measurement of the heat transport enhancement with respect to the non-rotating case as function of the rotation rate (Zhong et al. 2009 Stevens et al. 2009). The figure shows that, depending on the rotation rate, three different regimes can be identified. Regime I (weak rotation), where no heat transport enhancement is observed, regime II (moderate rotation), where a strong heat transport enhancement is found, and regime III (strong rotation), where the heat transport starts to decrease. We note that the division between regime I and regime II is obvious as there is a bifurcation (Zhong et al. 2009, Stevens et al. 2009, Weiss et al. 2010). Furthermore, we note that the log plot in figure 1 makes the transition from regime II to regime III look more 
sudden than it actually is. Zhong et al. (2009) and Stevens et al. (2009) have shown that the position of the onset does not depend on $R a$ and $P r$. However, the position of the maximum heat transfer enhancement shifts towards lower $1 / R o$ when $R a$ is increased or $P r$ is decreased. In addition, the maximum heat transfer enhancement decreases with increasing $R a$ and decreasing $P r$. For a detailed discussion on the influence of $R a$ and $P r$ we refer the reader to Zhong et al. (2009) and Stevens et al. (2010c). Flow visualization experiments (Boubnov \& Golitsyn 1990) Kunnen et al. |2010a) and the analysis of the flow structures obtained in numerical simulations (Stevens et al. 2009; Kunnen et al. $2010 a$ ) have confirmed that this division of regimes coincides with changes observed in the flow patterns and flow characteristics.

In regime I $(1 / R o \lesssim 0.5)$ the large scale circulation (LSC), typical for non-rotating $\mathrm{RB}$ convection, is still present, because the Coriolis force is too weak to overcome the buoyancy force that causes the LSC. Stevens et al. (2009) showed that there is a sharp transition to the regime where rotational effects become important, while at the same time the strength of the LSC is decreasing (Kunnen et al. 2008a). Zhong \& Ahlers (2010) experimentally found that the time-averaged LSC amplitudes decrease strongly at the transition from regime I to regime II, see figure 13 of their paper. In regime II $(0.5 \lesssim 1 / R o \lesssim 6.67)$ the LSC is replaced by predominantly vertically oriented vortical columns as the dominant flow structures and a large increase in the heat transport is observed.

This enhanced heat transport has been ascribed to Ekman pumping (Rossby 1969 Julien et al. |1996a, Vorobieff \& Ecke 2002, Kunnen et al. 2008a; King et al. 2009; Stevens et al. 2009). The effect of Ekman pumping, and thus the observed heat transfer enhancement, depends strongly on the $R a$ - and Pr-values (Zhong et al. 2009, Stevens et al. $2010 \mathrm{c})$. When the rotation rate is increased further a large decrease in the heat transport is observed, because the vertical velocity fluctuations are suppressed due to the rotation (Kunnen et al. $2008 b, 2010 a)$. We will call this regime III.

In this paper we address the question of how these different regimes can be identified from measurements of the azimuthal wall temperature distribution and the vertical temperature gradient along the sidewall, with probes that are embedded in the sidewall of a RB convection cell. This method of sidewall temperature measurements has been introduced by Brown et al. $(2005 b)$ and a validation of this method is described in the second paragraph of Section 2 of Brown \& Ahlers (2006b). Recently, Zhong \& Ahlers (2010) have extensively studied the properties of the LSC in RRB convection in aspect ratio $\Gamma=1$ experiments using this method. These measurements covered the $R a$ number range $3 \times 10^{8} \lesssim R a \lesssim 2 \times 10^{10}$, the $\operatorname{Pr}$ number range $3.0 \lesssim \operatorname{Pr} \lesssim 6.4$, and the $1 / R o$ number range $0 \lesssim 1 / R_{o} \lesssim 20$. In these measurements detailed statistics about the thermal LSC amplitude (i.e. the amplitude of the cosine fit to the azimuthal temperature profile at the sidewall), the LSC orientation over time, the temperature gradient along the sidewall, the retrograde rotation of the LSC, the frequency of cessations, etc., over this wide parameter range were determined.

This paper is organized as follows. The experimental setup that has been built at the Fluid Dynamics Laboratory at Eindhoven University of Technology is discussed in section 2. The design of this setup is closely based on the Santa Barbara design (Brown et al. 2005a). Subsequently we will present some experimental results on the properties of the azimuthal temperature profiles in section 3. For a much more detailed set of experimental results on the properties of the azimuthal temperature profiles we refer to the paper of Zhong \& Ahlers (2010). In Section 4 we compare the experimental data with the azimuthal temperature and vertical-velocity profiles close to the sidewall found in direct numerical simulations (DNS). In order to explain the sidewall temperature measurements 


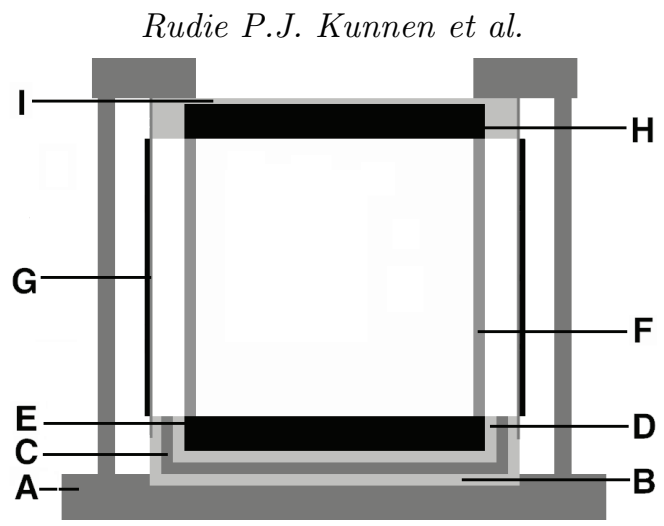

Figure 2. Schematic diagram (not to scale) of the Eindhoven RB apparatus. From bottom to top the figure shows the support plate (A), an insulation layer (B), the bottom adiabatic shield (C), an insulation layer (D), the bottom copper plate (E), the Plexiglas sidewall (F), the adiabatic side shield $(\mathrm{G})$, the top copper plate $(\mathrm{H})$, and the Plexiglas top plate $(\mathrm{I})$. See further details in the text.

we study the azimuthally averaged flow profiles obtained from DNS. The analysis presented in section 5 reveals that the sidewall temperature measurements, particularly the presence of the vertical wall temperature gradients in regimes II and III (Zhong \& Ahlers 2010), can be explained by the presence of Stewartson layers that are formed along the sidewall.

\section{Experimental setup}

Based on the setup described by Brown et al. (2005a) we have built a new RB setup in Eindhoven, which is suitable for high precision heat transport measurements. The convection cell has a diameter $D$ and height $L$ of $250 \mathrm{~mm}$, making the aspect ratio $\Gamma=D / L=1.0$. The modular design of the setup provides the ability to perform measurements at different aspect ratios as well, although this paper will only describe the $\Gamma=1$ setup in detail. During rotating experiments the $\mathrm{RB}$ cell is placed on the Eindhoven Rotating Table Facility (RTF) (for details, see van Bokhoven 2007). A rotating connection is available for coolant fluid, with separated in and out flow tubes. The rotation rate $\Omega$ of the RTF can be controlled from 0 to $10 \mathrm{rad} / \mathrm{s}$ with a resolution of $0.001 \mathrm{rad} / \mathrm{s}$ and a relative accuracy of $0.5 \%$. The rotation rates were kept below $\Omega=1.57 \mathrm{rad} / \mathrm{s}$, yielding Froude numbers $F r=\Omega^{2}(D / 2) / g$ less than 0.03 for all runs and much smaller for most. This means that the effect of centrifugal forces can be considered negligible.

A schematic diagram of the RB cell is shown in figure 2. From bottom to top, we first find the support plate $A$ (400 $\times 400 \mathrm{~mm}$ aluminium), used to mount the setup on the $\mathrm{RTF}$ and to align the rotational axes of the table and the convection cell so that the offset of the cylinder axis and the rotation axis is less than $0.1 \mathrm{~mm}$. Next, part $B$ is a $10 \mathrm{~mm}$ insulation layer to prevent heat loss of the bottom adiabatic shield $C(310 \mathrm{~mm}$ outer diameter, $10 \mathrm{~mm}$ thick aluminium) to the support plate. The shield is fitted with a $250 \mathrm{~W}$ heater to actively control its temperature. Inside this adiabatic shield, part $D$ is another $10 \mathrm{~mm}$ insulation layer, followed by the copper bottom plate $E$ (270 $\mathrm{mm}$ outer diameter, $30 \mathrm{~mm}$ thick) of the convection cell. The back of the bottom plate is covered uniformly with two double spiral grooves of $2 \mathrm{~mm}$ depth, $2 \mathrm{~mm}$ width and $6 \mathrm{~mm}$ spacing. Two $4 \mathrm{~m}, 12.3 \Omega$ resistance wires are epoxied into these grooves. From the back of the plate, five small holes (one at the centre, two at a radius of $98 \mathrm{~mm}$ and two at $100 \mathrm{~mm}$, i.e. in the space that is available between the heater wires that are placed inside the 
plate) are drilled to within $0.7 \mathrm{~mm}$ of its top surface and thermistors are mounted in these holes.

On top of the bottom plate, the Plexiglas sidewall $F$ with an inner diameter of $250 \mathrm{~mm}$ is placed. The thickness of the Plexiglas sidewall is $10 \mathrm{~mm}$ to provide enough strength in strongly RRB experiments. A rubber O-ring seals the interface between the bottom plate and the sidewall and prevents any leakage of fluid. The sidewall contains a fluid inlet near the bottom plate and, at opposite angular position, an outlet adjacent to the top plate. Care has been taken to avoid the entrapment of air in the convection cell, both when filling the cell and when heating the fluid. The sidewall is surrounded by an adiabatic side shield $G$ made of two $3 \mathrm{~mm}$ thick aluminium plates. The shield is actively temperature controlled, ensuring that its temperature is always close to the mean temperature of the system. Additional insulation is present between the Plexiglas side wall and the aluminium side shield.

The top of the convection cell is formed by the copper top plate $H$, which has similar dimensions as the bottom plate. The top plate contains a double spiral water channel of $8 \mathrm{~mm}$ width, $26 \mathrm{~mm}$ depth and $25 \mathrm{~mm}$ spacing. The water, coming from a refrigerated circulator (Thermo Scientific HAAKE DC50-K41) at $12.5 \mathrm{~L} / \mathrm{min}$, cools the top plate down to the desired temperature. From the top of the plate, five small holes (one at the centre, four at a radius of $100 \mathrm{~mm}$ ) were drilled to within $0.7 \mathrm{~mm}$ of the copperfluid interface and thermistors are mounted in these holes. The top plate is covered by a Plexiglas top $I$ and the water channel is sealed with a rubber ring. The visual access provides a way to check the proper operation of the cooling system. An aluminium construction ring is connected to the upper side of the top plate. It is supported by 6 stainless steel support poles. With these poles, the RB cell can be fixed to achieve a fully watertight connection between the plates and the sidewall. To decrease the effects of air convection near the convection cell, the entire construction is placed in a wooden box covered with a $40 \mathrm{~mm}$ insulating layer on the inside.

The apparatus contains 40 thermistors, which were calibrated simultaneously in a separate apparatus against a laboratory standard. During the calibration procedure all thermistors and the laboratory standard are placed in an extremely well temperaturecontrolled batch (temperature differences less than $0.002 \mathrm{~K}$ ) to determine the resistance at a set of known temperatures. From this calibration data fits were made with 5 -th order logarithmic polynomials to calculate temperature values from 3-wire resistance measurements. Deviations from these fits are generally less than $0.001 \mathrm{~K}$. During the calibration procedure all thermistors are connected to the same software and hardware as in the real experiments. 24 of these thermistors are placed in the sidewall, forming 3 rings of 8 equally spaced sensors at heights $0.25 \mathrm{~L}, 0.5 \mathrm{~L}$ and $0.75 \mathrm{~L}$. Both the top and bottom plate contain 5 sensors to monitor the temperature of the plate. The remaining sensors are used to control the temperature of the bottom adiabatic shield and of the insulation around the Plexiglas sidewall. Readings of all thermometer resistances and of the bottom plate heater current and heater voltage were taken every second. The top (bottom) temperature $T_{t}\left(T_{b}\right)$ was set equal to the area average of the five thermometers embedded in the top (bottom) plate. For any given data point, measurements over typically the first four hours were discarded to avoid transients, and data taken over an additional period of at least another eight hours were averaged to get the heat-current density $Q$, and the temperatures $T_{b}$ and $T_{t}$. Because of the imperfect temperature uniformity of the bottom shield there was a small parasitic heat loss from the bottom plate of about $0.1 \mathrm{~W}$, which was determined by measuring the required power to keep the bottom and top plate at $40^{\circ} \mathrm{C}$, a measurement which takes two days. For each measurement this parasitic heat loss was subtracted from the measured power. 


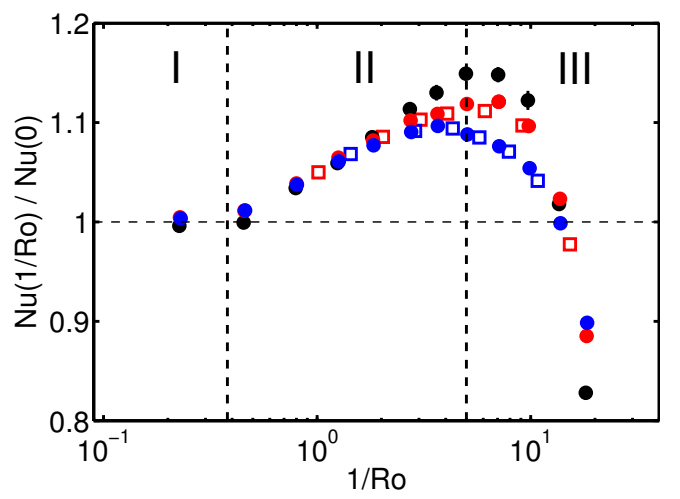

Figure 3 . The ratio of the Nusselt number $N u(1 / R o)$ in the presence of rotation to $N u(0)$ for $\operatorname{Pr}=4.38\left(T_{m}=40.00^{\circ} \mathrm{C}\right)$ and $\Gamma=1$. The solid circles are the data obtained in the Eindhoven RB setup and the open squares are the corresponding data obtained in Santa Barbara (Zhong et al. 2009, Stevens et al. 2009). Black solid circles: $R a=2.99 \times 10^{8}(\Delta=0.50 \mathrm{~K})$. Red solid circles: $R a=5.88 \times 10^{\circ}(\Delta=1.00 \mathrm{~K})$. Blue solid circles: $R a=1.16 \times 10^{9}(\Delta=2.00 \mathrm{~K})$. Red open squares: $R a=5.6 \times 10^{8}(\Delta=1.00 \mathrm{~K}$, run E4 in Zhong \& Ahlers 2010). Blue open squares: $R a=1.2 \times 10^{9}(\Delta=2.00 \mathrm{~K}$, run E5 in Zhong \& Ahlers 2010).

The measurements have been verified against the experiments of Funfschilling et al. (2005), Ahlers et al. (2009) and Stevens et al. (2009). For the present measurements the difference is in the order of $1 \%$, well within the differences observed between different RB setups (Ahlers et al. 2009). In figure 3 we show the heat transfer enhancement $N u(\Omega) / N u(0)$ as function of the rotation rate $1 / R o$ for $R a=2.99 \times 10^{8}, R a=5.88 \times 10^{8}$ and $R a=1.16 \times 10^{9}$ at $\operatorname{Pr}=4.38$, which corresponds with a mean temperature of the fluid of $40.00^{\circ} \mathrm{C}$, and compare them with similar heat transport measurements reported in the literature (Zhong et al. 2009 ; Stevens et al. 2009$)$. We find that the two datasets involving rotation also agree within $1 \%$, which can be considered a very good agreement.

\section{Experimental sidewall measurements}

In $\mathrm{RB}$ experiments it is common to determine the flow properties by analyzing the azimuthal wall temperature profile obtained by thermistors that are embedded in the sidewall (Ahlers et al. 2009) and in our setup we do the same. Following Stevens et al. $(2010 b)$ we define the relative LSC strength at midheight $\left(\bar{S}_{m}\right)$, based on the energy in the different modes of the azimuthal temperature profile, as

$$
\bar{S}_{m}=\max \left(\left(\frac{\sum_{t_{b}}^{t_{e}} E_{1}}{\sum_{t_{b}}^{t_{e}} E_{t o t}}-\frac{1}{N}\right) /\left(1-\frac{1}{N}\right), 0\right) .
$$

Here $\sum_{t_{b}}^{t_{e}} E_{1}$ indicates the sum of the energy in the first Fourier mode over time, i.e. from the beginning of the simulation $t=t_{b}$ to the end of the simulation $t=t_{e}, \sum_{t_{b}}^{t_{e}} E_{t o t}$ the sum of the total energy in all Fourier modes over time, and $N$ the total number of Fourier modes that can be determined. The relative LSC strength $\bar{S}_{m}$ always has a value between 0 and 1 ; here 1 indicates that the azimuthal profile is a pure cosine profile, which is a signature of the LSC according to Brown \& Ahlers $(2006 b)$, and 0 indicates that the magnitude of the cosine mode is equal to (or weaker than) the value expected from a random noise signal. Hence $\bar{S}_{m} \gg 0.5$ indicates that a cosine fit on average is a reasonable approximation of the data, as then most energy in the signal resides in the first Fourier mode. In contrast, $\bar{S}_{m} \ll 0.5$ indicates that most energy resides in the higher 
Fourier modes. Hence, we consider the LSC as dominant once $\bar{S}_{m} \gg 0.5$ at midheight. A small value of $\bar{S}_{m}$ indicates that no single LSC is found, implying the existence of either multiple rolls or, in the case of rotating Rayleigh-Bénard (RRB) convection, vertically aligned vortices.

Figure 4(a) shows the magnitude of the different Fourier modes of the azimuthal wall temperature profile for the experiments at $R a=1.16 \times 10^{9}$ with $\operatorname{Pr}=4.38$ based on the data of eight equally spaced thermistors placed inside the sidewall (located at $z=0.5 L$ ). The corresponding relative LSC strength is given in panel $\mathrm{b}$. The figure clearly shows that the relative LSC strength is large in regime I, which indicates the presence of a LSC. However, for higher rotation rates, i.e. regime II, the relative LSC strength decreases because vertically aligned vortices become the dominant feature of the flow. For this flow one expects a random azimuthal temperature profile at midheight, which is confirmed by the low relative LSC strength $S_{m}$ in regime II and III, see figure 4 (b).

Extensive sidewall temperature gradient measurements for non-rotating RB convection were done by Brown \& Ahlers (2007) and for RRB convection by Zhong \& Ahlers (2010), see their figures 10 and 11. When the LSC is the dominant feature of the flow the vertical temperature gradient at the sidewall is mainly due to plumes that travel close to the sidewall with the LSC. Thus one would expect that the temperature gradient along the sidewall should decrease in regime II as the LSC disappears there. However figure 10 of Zhong \& Ahlers (2010) shows that the temperature gradient along the sidewall even increases in regime II and III. This nonzero temperature gradient along the sidewall in regime II and III is caused by the secondary flow that will be discussed in section 5 . Further interesting information about the LSC can be found in figure 13 of Zhong \& Ahlers (2010). The figure shows that the temperature amplitude of the LSC, i.e. the strength of the first Fourier mode, starts to decrease at $1 / R o \approx 0.41$ and that the LSC ceases to contribute significantly for $1 / R o \gtrsim 0.8$. Thus our experimental results in figure 4 are in good agreement with the results of Zhong \& Ahlers (2010). It is interesting to note that the measurements of Zhong \& Ahlers (2010) reveal an increase in the LSC strength between $1 / R o=0$ and $1 / R o \approx 0.41$. In our data this effect is not visible, since we do not have enough measurement points in regime I. As is discussed by Zhong \& Ahlers (2010) there is no well-accepted theoretical explanation for this phenomenon at the moment.

\section{Numerical study of temperature and vertical-velocity profiles close to the sidewall}

We shall now consider the results of DNS of RRB convection performed at various Ro values for $R a=2.73 \times 10^{8}$ and $\operatorname{Pr}=6.26$ (see details in Zhong et al. 2009) and for $R a=1.00 \times 10^{9}$ and $\operatorname{Pr}=6.4$ (see details in Kunnen et al. 2010a), which are in the same parameter regime as the experiments discussed above. In the simulations we solved the three-dimensional Navier-Stokes equations within the Boussinesq approximation in a cylindrical cell with $\Gamma=1$ with no-slip boundary conditions at all walls, a uniform temperature at the horizontal plates, and an adiabatic sidewall. For further details about the numerical code we refer to Verzicco \& Orlandi (1996) and Verzicco \& Camussi (1997, 2003). In all simulations we calculate the azimuthal averages of the three velocity components and the temperature. Furthermore, in the simulations at $R a=2.73 \times 10^{8}$ we placed 32 azimuthally equally spaced numerical probes that provide simultaneous pointwise measurements of the temperature and the three velocity components $u_{r}, u_{\phi}, u_{z}$ in the radial, azimuthal, and vertical directions $r, \phi$ and $z$, respectively, at the heights $0.25 \mathrm{~L}, 0.50 \mathrm{~L}$, and $0.75 \mathrm{~L}$ and a distances $0.45 \mathrm{~L}$ from the cylinder axis. The azimuthal 


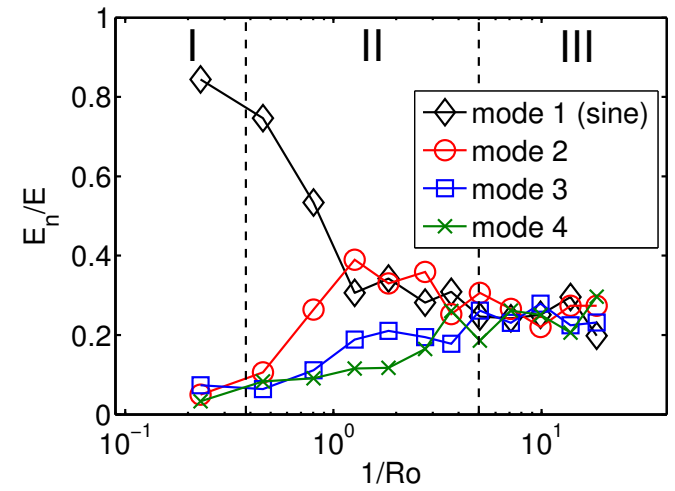

(a)

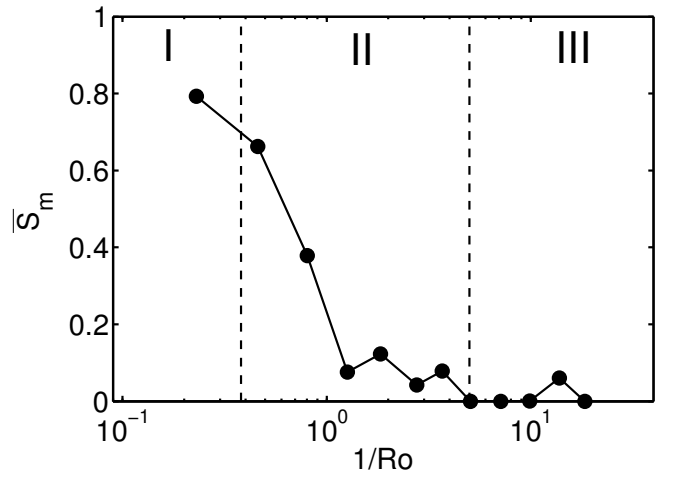

(b)

Figure 4. Experimental results on the magnitude of the different Fourier modes and $S_{m}$ for $R a=1.16 \times 10^{9}$ and $\operatorname{Pr}=4.38$ based on the data of 8 azimuthally equally spaced probes at $z=0.50 L$. a) The energy in the different Fourier modes of the azimuthal wall temperature profile normalized by the energy present in all Fourier modes as function of $1 / R o$. b) The corresponding relative LSC strength $S_{m}$. The two dashed vertical lines in both panels indicate the transitions between regimes I and II, and between II and III, respectively.

temperature and vertical-velocity profiles measured by the probes are analyzed in the same way as the experimental data.

Figure 5 shows the magnitude of the different Fourier modes of the azimuthal verticalvelocity profiles for $R a=2.73 \times 10^{8}$ and $\operatorname{Pr}=6.26$ based on the data of 8,16 , and 32 equally spaced probes. The result in panel (a) agrees well with the result obtained by Kunnen et al. (2008a). Furthermore, a comparison of the relative energy in the different Fourier modes based on the data of 8,16 , and 32 probes, see figure 5 reveals that 8 probes are insufficient to capture all flow characteristics. Since the results based on the data of 16 and 32 probes are very similar, we assume that 16 probes should be sufficient to capture all relevant features of the azimuthal profiles.

Fortunately, the data of 8 equally spaced thermistors are already sufficient to reliably calculate $S_{m}$ for non-rotating RB convection, see Stevens et al. $(2010 b)$. To confirm this observation for the RRB case (and validate the experimental results with 8 probes discussed in section 3 we therefore calculate $S_{m}$ based on the data of 8,16 , and 32 equally spaced probes. The result is given in figure 5(d), which shows that the curves for the relative LSC strength almost collapse for the three cases. Moreover, the numerical data are in good agreement with the experimental result shown in figure 4 , including the large decrease of $S_{m}$ at the transition between regimes I and II. The small value of the relative LSC strength $\left(S_{m} \ll 0.5\right)$ suggests the absence of the LSC and that the azimuthal temperature profile at midheight becomes random in regime II and III. This is confirmed by a three-dimensional visualization of the flow, see figure 5 of Zhong et al. (2009), where it is shown that in regime II vertically aligned vortices are the dominant feature of the flow. We note that figure 5 shows a continuous decrease of the relative LSC strength, which is in disagreement with the measurements of Zhong \& Ahlers (2010) that show a small increase in the temperature amplitude of the LSC between $1 / R o=0$ and $1 / R o \approx 0.41$. This difference might be caused by the slightly different monitoring locations (inside the sidewall in the experiments and at the radial position $0.45 \mathrm{~L}$ in the numerical simulations). 


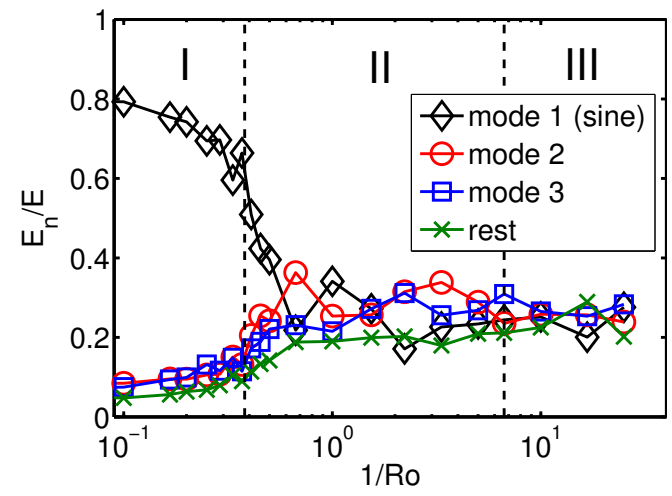

(a)

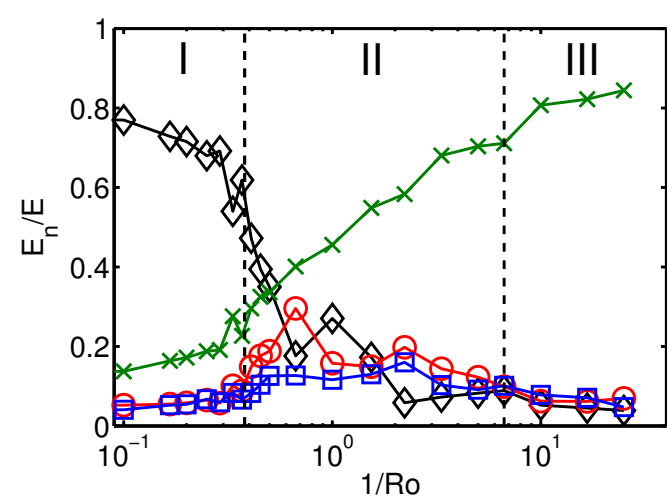

(c)

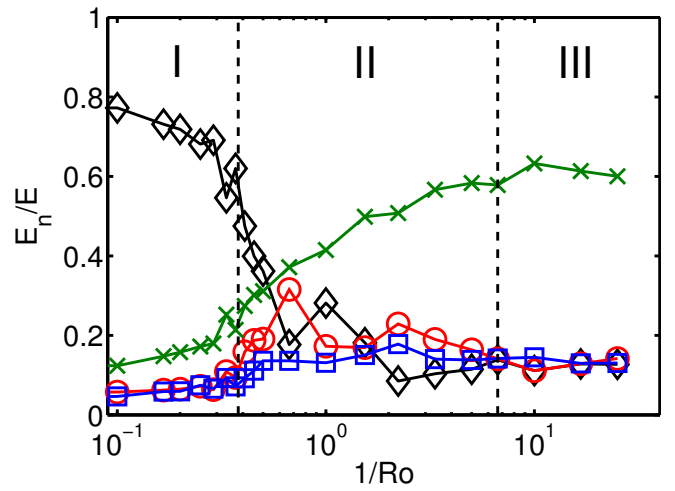

(b)

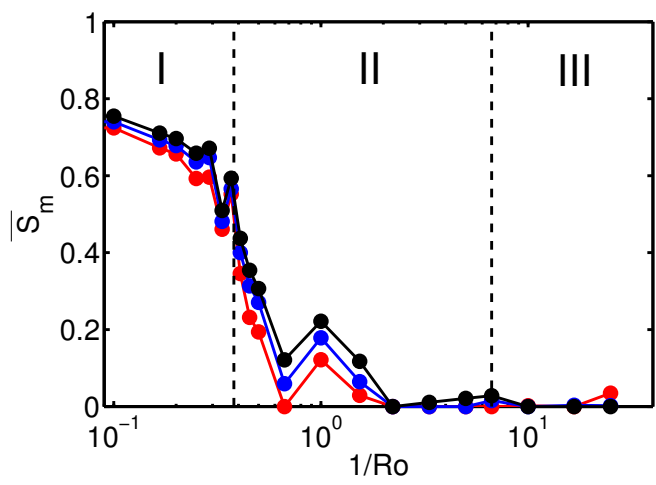

(d)

Figure 5. Results from DNS on the magnitude of the different Fourier modes and $S_{m}$ for $R a=2.73 \times 10^{8}$ and $\operatorname{Pr}=6.26$ based on the data of the numerical probes at $z=0.50 \mathrm{~L}$. Panels (a), (b), and (c) show the energy in the different Fourier modes of the azimuthal temperature profile normalized by the energy present in all Fourier modes based on the data of 8, 16, and 32 equally spaced probes, respectively. The black, red, blue, and dark green lines indicate the energy in the first, second, third, and the additional modes, respectively. Note that when the data of 8 probes are used a lot of information about the higher modes is lost. Panel $\mathrm{d}$ shows the corresponding relative LSC strength based on the data of 8 (red), 16 (blue), and 32 (black) equally spaced probes. The two dashed vertical lines indicate the transitions between regimes I and II, and between II and III, respectively.

\section{The role of Ekman and Stewartson boundary layers}

In this section we will first discuss the flow characteristics for regimes I and II and sketch the influence of background rotation on the mean flow in general terms, and the role of the Ekman and Stewartson boundary layers is emphasized. Subsequently, we provide physical explanations for the structure of the mean flow as observed for regime II. Finally, we discuss the implications for the mean temperature gradient at the sidewall of the convection cell.

\subsection{Description of the flow characteristics}

In figure 6 we show the azimuthally averaged temperature and velocity components $u_{z}$, $u_{\phi}$ and $u_{r}$ for three typical rotation regimes: (the top row) $1 / R o=0$ (no rotation, regime I), (the middle row) $1 / R o=0.35$ (weak rotation, regime I) and (the bottom 
row) $1 / R o=2.78$ (moderate rotation, regime II) at $R a=1 \times 10^{9}$ and $\operatorname{Pr}=6.4$. The left-hand side of each picture $(r=0)$ represents the position of the cylinder axis (dashdotted line), while the right-hand side $(r / L=0.5)$ corresponds with the sidewall. In addition to the azimuthal averaginging, they have also been averaged in time for more than one hundred large-eddy turnover times to find the mean circulation hidden under the turbulent fluctuations. A close-up of the thermal and flow structure in the bottom corner of the tank for the case $1 / R o=2.78$ (see figure 6c) is shown in figure 7 . We note that the averaged profiles for lower $R a$ values are similar to the ones presented in figure 6]

For the non-rotating case $(1 / R o=0)$, shown in $6(\mathrm{a})$, we observe the signature of the LSC. When looking at a vertical cross-section of the domain, the cross-sectional plane aligned with the LSC, the mean circulation has an elliptic shape with its major axis oriented at some angle with the cylinder axis (see the sketch in figure 8, and also in Qiu \& Tong (2001)). It is due to this mean tilt of the elliptic LSC, combined with the rotation sense as shown in figure 8, that the azimuthal average of this flow is not zero; especially upward and downward motions are separated in these azimuthally averaged plots. In the top half upward motion (positive $u_{z}$ ) is found near the cylinder axis, while the downward flow (negative $u_{z}$ ) is strongest near the sidewall. The opposite situation is found for $u_{z}$ in the bottom half. The azimuthal velocity $u_{\phi}$ does not show a well-defined mean profile, just some small fluctuations. The averaged radial velocity $u_{r}$ has maximal values near the intersections of the horizontal plates with the sidewall, consistent with the averaged vertical velocity $u_{z}$ near these regions. Note also the weak radially inward flow near the sidewall around midheight.

When a small rotation is introduced $[1 / R o=0.35$, figure 6(b)], a first observation is that the mean velocities become smaller (except the averaged azimuthal velocity $u_{\phi}$ which grows due to organization of the azimuthal flow). The vertical velocity $u_{z}$ still has the dominant structuring due to the LSC, but especially near the cylinder axis some disturbances appear. For the azimuthal velocity a well-defined structure emerges due to the azimuthal averaging. The Coriolis force, deviating the parts of the LSC with mean horizontal velocity to the right with respect to the local direction of the (mean) horizontal flow, is driving the organization of an average azimuthal flow. The blue regions near the corners in the plot of the azimuthal velocity $u_{\phi}$ represent the anticyclonic motion induced by the mean horizontal outward flow of the LSC (near the bottom and top plates, see figure 8). The sketch of the LSC motion shows that the up (down) going plumes are first traveling straight up (down), before the radial inward flow of the LSC sets in. The cyclonic motion that is observed in the central sidewall region of the azimuthally averaged azimuthal velocity is due to the radial inward flow of the LSC in the top (bottom) half of the cell for the upward (downward) branch of the LSC, see figure 8. This radial inward flow of the LSC results in a spin-up, i.e. in cyclonic azimuthal motion, by the action of the Coriolis force. This mechanism is equivalent to conservation of angular momentum: radial inward motion $\left(u_{r}<0\right)$ of fluid parcels results in an increase of $u_{\phi}$, i.e. in cyclonic motion. Because this radial inward flow is in the top (bottom) part of the sample for the up (down) going plumes this results, after azimuthal averaging, in one central sidewall region in which a cyclonic motion is found. This means that this particular snapshot, cannot be used for interpretation of the movement of individual plumes traveling from bottom to top and the other way around. However, in this very schematic picture it does seem to be consistent with experimental measurements of the anticyclonic motion of the LSC (Hart et al. 2002, Brown \& Ahlers 2006a; Kunnen et al. 2008a; Zhong \& Ahlers 2010 ) as both upgoing and downgoing plumes get anticyclonic deflection by the mean 
anticyclonic azimuthal flow near the plates and any effect of mean cyclonic flow becomes effective only for $z \gtrsim 2 / 3 H$ (for upgoing plumes) and $z \lesssim H / 3$ (for downgoing plumes).

At much higher rotation rates, i.e. for larger $1 / R o$-values, the LSC no longer exists, and the secondary circulation takes on a different appearance. It is obvious from the velocity plots that relatively large mean velocities occur in thin regions near the solid container walls. A division of the domain into several regions is appropriate, being the bulk interior domain, the Ekman layers at the horizontal plates, and the Stewartson layer at the sidewall. The Ekman layers have a (non-dimensional) thickness $\delta_{E}=E k^{1 / 2}$ (with the Ekman number defined as $E k=\nu /\left(\Omega L^{2}\right)$, representing an inverse Reynolds number based on the length scale $L$ and velocity scale $L \Omega$ ). The Stewartson layer at the sidewall has a sandwich structure consisting of a thicker outer layer of non-dimensional thickness $\delta_{S, 1 / 4}=E k^{1 / 4}$ and a thinner inner layer of non-dimensional thickness $\delta_{S, 1 / 3}=E k^{1 / 3}$ (see e.g. Stewartson 1957, 1966, Moore \& Saffman 1969, van Heijst 1983, 1984, 1986). The dashed lines in figure 6(c) and figure 7 indicate these typical layer thicknesses. In figure 7 the bottom right-hand corner is magnified for the temperature (top panel) and each of the velocity components, so that the various boundary layers are more easily recognized. Note that since the secondary circulation associated with the Ekman and Stewartson layers is weak, the mean velocities $u_{r}$ and $u_{z}$ in the bulk are very small and therefore hardly noticeable, as is confirmed by the simulation results. It should be emphasized that the secondary circulation found here is considerably different from that found by Hart \& Olsen (1999) or that of Homsy \& Hudson (1969), both driven by centrifugal buoyancy. In both these works the up-down symmetry is broken due to centrifugal accelerations having opposite directions near the plates, viz. cold fluid near the top plate accelerates radially outward while hot fluid near the bottom plate goes radially inward. Here we disregard centrifugal acceleration and up-down symmetry is preserved. It must also be stated that the aforementioned circulation is only of secondary magnitude. The still-turbulent field of the vortical plumes is dominant.

The most remarkable change is observed in the distribution of the azimuthal velocity $u_{\phi}$ : in the bulk of the domain an anticyclonic flow $\left(u_{\phi}<0\right.$, indicated by blue in figure 6 ) is present, while a thin band of cyclonic flow $\left(u_{\phi}>0\right.$, indicated by red) is visible only close to the sidewall. Simulations for the parameter range $0.35 \lesssim 1 / R o \lesssim 2.78$ (not shown here) revealed that for increasing rotation rates (increasing $1 /$ Ro-values) the cyclonic flow region becomes confined to a region near the sidewall of increasingly smaller size. These observations suggest that in this Ro-regime the Ekman and Stewartson layers play an essential role in the mean secondary circulation, even though this circulation is relatively weak and even continuously disturbed by the non-steady thermally driven turbulent plumes that are present throughout the flow domain.

\subsection{Analysis secondary circulation regime II}

The feature of the weak anticyclonic swirl flow in the bulk of the domain in combination with a region of cyclonic swirling motion near the cylinder wall (the $u_{\phi}$-plot in figure 6(c)) is remarkable. Although the exact mechanism causing this bulk motion is an open question, we offer a possible explanation as follows. The heated fluid rising upward from the bottom plate and the cooled fluid flowing downward from the top plate result in a radially outward mean flow in the bulk of the domain, as sketched in the $(r, z)$ plane in figure 9(a). Although this radial motion is relatively weak, conservation of angular momentum ( $\sim r V$, with $V=\Omega r+u_{\phi}$ the absolute azimuthal velocity) implies that the absolute swirl velocity of fluid parcels will decrease, i.e. in the co-rotating frame this fluid will acquire an anticyclonic azimuthal motion $\left(u_{\phi}<0\right)$. This radial outflow in the bulk of the domain is of such small magnitude that it leaves no strong trace in the azimuthally 
12
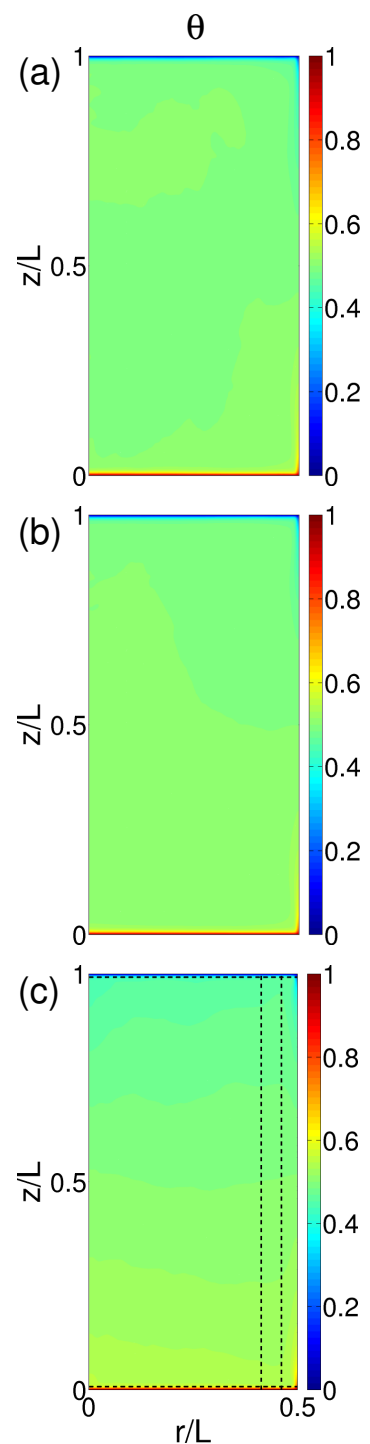

Rudie P.J. Kunnen et al.
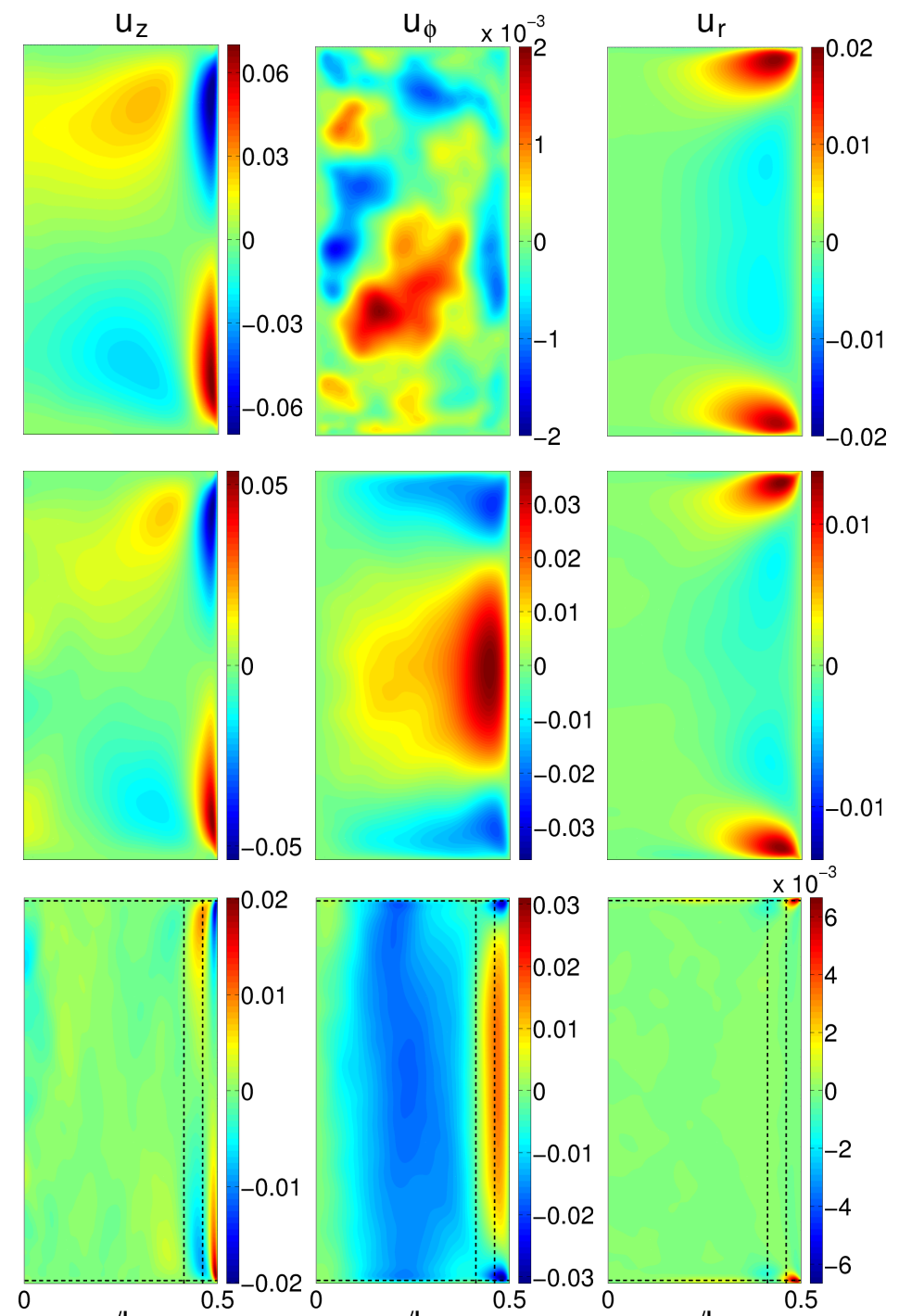

$\mathrm{r} / \mathrm{L}$

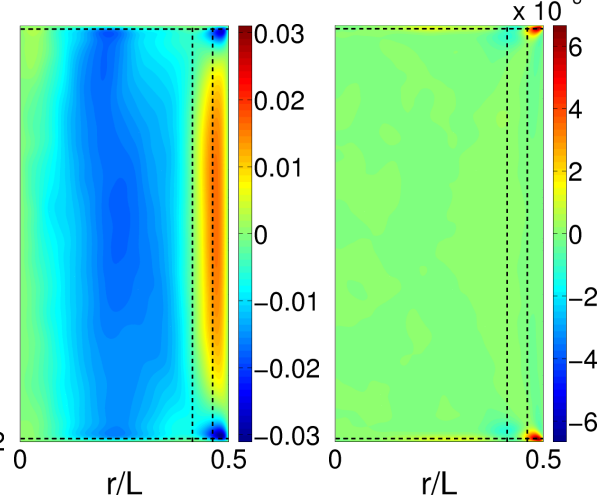

$\mathrm{r} / \mathrm{L}$

$\mathrm{r} / \mathrm{L}$

FigURE 6 . The figures from left to right indicate temporal and azimuthal averages of temperature and the velocity components $u_{z}, u_{\phi}$, and $u_{r}$, respectively, for $R a=1.00 \times 10^{9}$ and $\operatorname{Pr}=6.4$. The rows from top to bottom indicate the results for (a) $1 / R o=0$, (b) $1 / R o=0.35$, and (c) $1 / R o=2.78$, respectively. The left-hand side of each picture is the cylinder axis $r=0$; the right-hand side corresponds to the sidewall $r / L=0.5$. The dashed lines in the pictures of the bottom row indicate typical boundary-layer thicknesses: $\delta_{E}=E k^{1 / 2}$ near the bottom and top plates, and $\delta_{S, 1 / 3}=E k^{1 / 3}$ and $\delta_{S, 1 / 4}=E k^{1 / 4}$ near the sidewall ( $\delta_{S, 1 / 3}$ is closest to the sidewall). Note that the azimuthally averaged azimuthal velocity for $1 / R o=0$ (a) is much smaller than for $1 / R o=0.35(\mathrm{~b})$ and $1 / R o=2.78(\mathrm{c})$.

averaged radial velocity $u_{r}$. Furthermore, the plots of averaged vertical velocity $u_{z}$ show no mean motion directed away from the horizontal plates: this transport is localised in the small thermal vortices that vanish after azimuthal averaging.

This picture of the flow is not complete, however, because a second mechanism is active simultaneously in the bulk of the domain: the spin-up process (Greenspan \& Howard 1963). This second process is driven by the Ekman layers (figure 9b): the bottom Ekman 

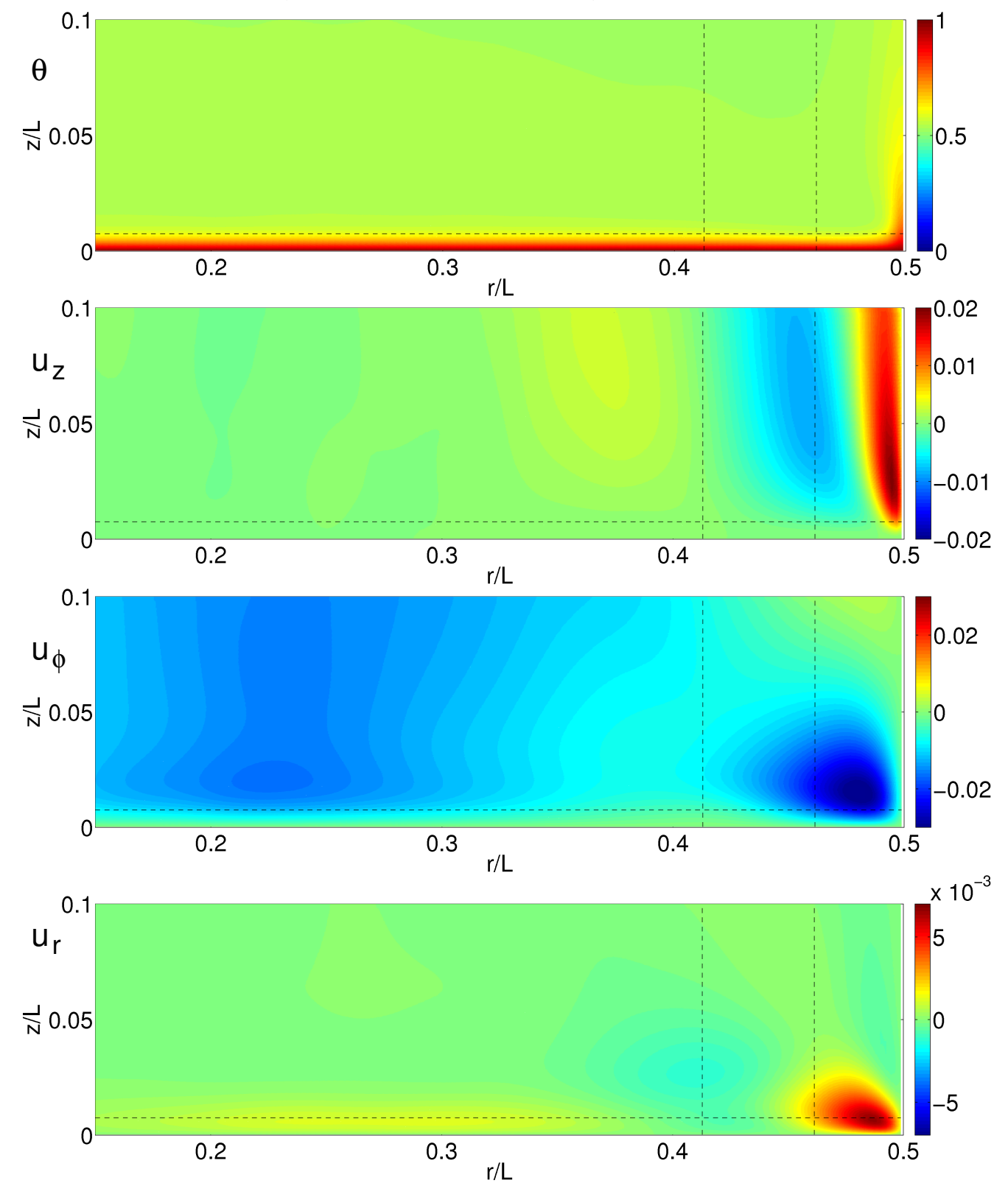

FiguRE 7. Close-up of the bottom-right corner of the figures shown in figure 6(c) $(1 / R o=2.78)$. The area displayed is $0.15<r / L<0.5 ; 0<z / L<0.1$. Color coding and dashed lines as in figure 6(c). The panels from top to bottom indicate the temporal and azimuthal averages of temperature and the velocity components $u_{z}, u_{\phi}$, and $u_{r}$, respectively.

layer imposes a suction velocity on the interior given by $u_{z}=\frac{1}{2} E k \omega_{I}$ in dimensionless form, with $\omega_{I}$ the vertical component of the rotation of the relative interior flow. To clarify, as $\omega_{I}$ is anticyclonic, the corresponding vertical velocity according to the suction condition is directed from the bulk into the Ekman layer. A similar suction velocity is imposed on the interior flow by the upper Ekman layer, also directed from the bulk to the boundary layer. There is thus a mean flow from the bulk into the Ekman layers. By 


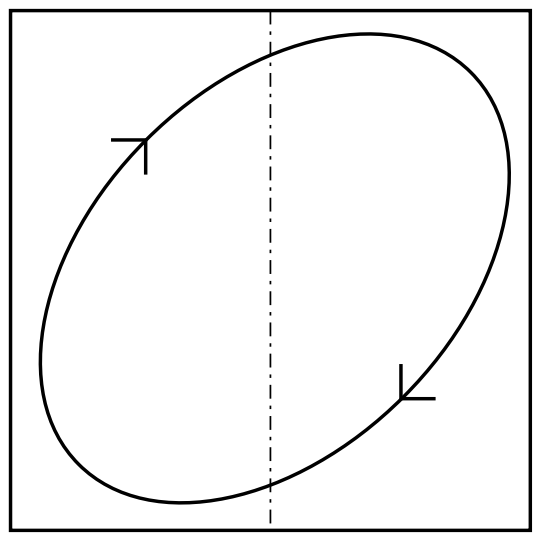

Figure 8. Schematic side view of the tilted LSC in the cylinder in the non-rotating or weakly rotating (regime I) case. The dash-dotted line is the axis of the cylinder.

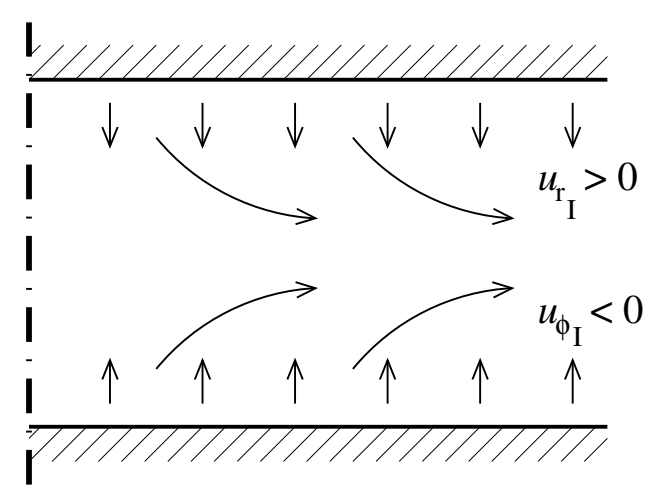

(a)

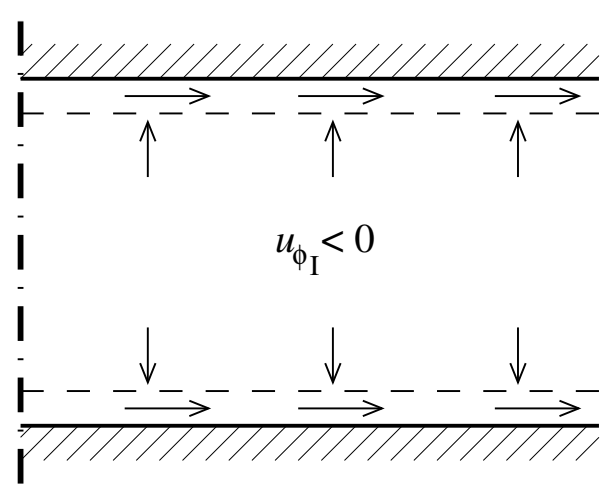

(b)

Figure 9. (a) Fluid motion due to thermal forcing. The heated (cooled) fluid rising upward (flowing downwards) from the bottom (top) plate result in a radially outward mean flow in the bulk of the domain. (b) Fluid motion due to the suction of the Ekman layers (spin up). The Ekman layers impose suction towards the plates. Mass conservation implies a radial outflow inside these layers. The dash-dotted line in both panels indicates the center of the cell (which coincides with the rotation axis).

conservation of mass, a radial outflow in the Ekman layers is needed to carry away the flux from the bulk into the Ekman layers. The radial outflow is easily identified in the averaged radial velocity plots due to the tiny radial cross-sectional area of the Ekman layer, leading to a relatively large mean velocity.

The radially outward transport through the Ekman layers is returned via a Stewartson boundary layer at the cylinder wall. This layer has a small mean radial inward transport into the interior distributed along its vertical extent. An indication of this radially inward transport is found in the $u_{r}$ plot of figure 7 at $(r, z) \approx(0.4,0.025)$. There is also an internal recirculation in this layer: Again, according to the principle of conservation of angular momentum $r V$, with $V=\Omega r+u_{\phi}$, the radial inward motion from the sidewall boundary layer in the bulk implies a change in the swirl velocity, which is reduced in magnitude and approaches zero.

It can be shown that the matching of the azimuthal velocity component $u_{\phi}(r=0.5 L)$ to 


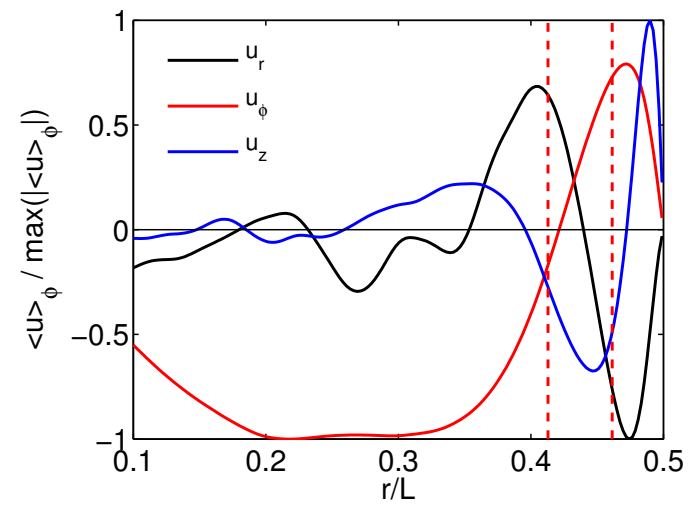

Figure 10. The azimuthally averaged velocity profiles at $z=0.25 L$ for $R a=1.00 \times 10^{9}$ and $\operatorname{Pr}=6.4$ for $1 / R o=2.78$. The azimuthal, radial, and axial velocity components are indicated in red, black, and blue, respectively.

the sidewall requires a Stewartson layer of typical thickness $E k^{1 / 4}$, in which this velocity is given by $u_{\phi}=-\frac{1}{L} u_{\phi}(r=0.5 L) \exp (\xi \sqrt{2 / L})$, with $L$ the height of the cylinder, and $\xi=$ $(r-0.5 L) E k^{-1 / 4}$ the stretched radial boundary-layer coordinate (Greenspan \& Howard 1963). When referring to this principal velocity component as being $\mathcal{O}(1)$, the vertical and radial velocity components in this layer are much smaller, viz. $\mathcal{O}\left(E k^{1 / 4}\right)$ and $\mathcal{O}\left(E k^{1 / 2}\right)$, respectively (in non-dimensional terms). The radial velocity is also correctly matched to the sidewall. However, matching of the vertical velocity to the cylinder wall requires the presence of a thinner $E k^{1 / 3}$ layer inside the $E k^{1 / 4}$ layer. The vertical matching implies a principal solution with a vertical velocity $u_{z} \sim \mathcal{O}\left(E k^{1 / 4}\right)$ within this layer. However, the entire velocity field in the $E k^{1 / 3}$ layer carries no net vertical flux; it is just an internal recirculation (Greenspan \& Howard 1963).

We will now describe the internal recirculation in more detail. Where the Stewartson $E k^{1 / 3}$ layer meets the Ekman layers, the vertical velocity has a singular structure (Moore \& Saffman 1969, van Heijst 1983, 1986): $u_{z} \sim \pm \delta(\eta)$ at $z=0$ and $L$, with $\eta=(r-$ $0.5 L) E k^{-1 / 3}$ the stretched radial coordinate in this Stewartson layer, and $\delta$ the Dirac delta function. Signs of these singular eruptions of the Ekman layer fluxes at $z=0$ and $z=L$ into the thinner Stewartson layer on the sidewall are clearly visible in the $u_{z}$-plot in figure 6(c), and for the case of the bottom corner in figure 7. Note that these vertical fluxes are positive and negative near the bottom and top Ekman layers, respectively. Weaker vertical velocities of opposite signs are observed in the thicker $E k^{1 / 4}$ layer, see figure 6(c). This oppositely signed vertical velocity next to the peak corresponds with the internal recirculation of the singular upward flux. Furthermore, the radial branches of the internal recirculation can be recognised in the $u_{\theta}$ plots. Close to the bottom and top plates there is strong radially outward flow in the thin Ekman boundary layers, which corresponds to strongly negative azimuthal velocity (corner regions near the sidewall of figures 6(c) and 7). Along the sidewall away from the plates there is radial inflow to close this recirculation, which, due to the much larger vertical extent, is of such small magnitude that it is not found in the plot of mean radial velocity. However, the radial inflow leaves its signature on the mean azimuthal velocity: radial inflow corresponds to positive $u_{\theta}$, which is observed as the orange band near the sidewall in the $u_{\theta}$ plot of figure 6 (c).

To further illustrate the secondary circulation we consider radial mean profiles of velocity. Figure 10 (a) shows the azimuthally averaged velocity profiles $u_{r}(r), u_{\phi}(r)$, and 


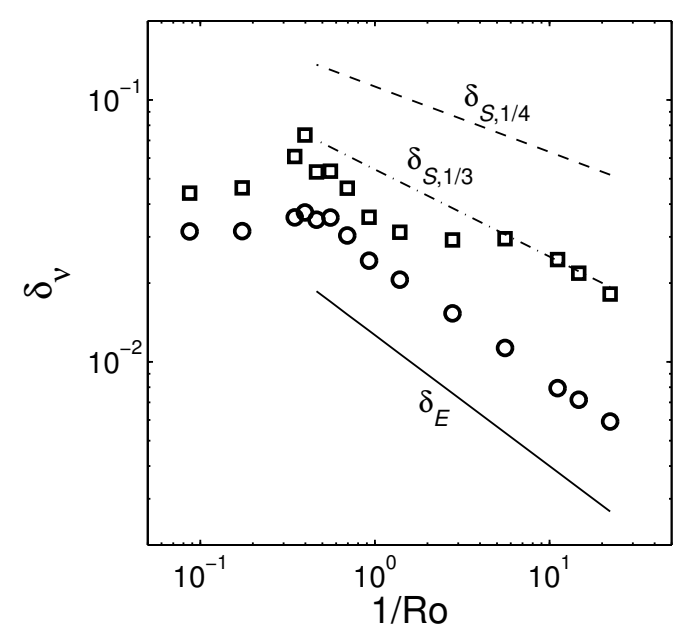

FiguRE 11. Boundary layer thicknesses for $R a=1.00 \times 10^{9}$ and $\operatorname{Pr}=6.4$. The dimensionless viscous boundary layer thickness near the plates is indicated by the circles. The dimensionless boundary layer thickness of the viscous boundary layers near the sidewall, i.e. the Stewartson layers, is indicated by the squares and follows the scaling of the inner Stewartson boundary layer thickness, i.e. $\delta_{S, 1 / 3}$. Adapted from Kunnen et al. (2010a).

$u_{z}(r)$ at $z=0.25 L$ according to a numerical simulation for $1 / R o=2.78$. The radial $u_{\phi}$-distribution clearly shows the presence of anticyclonic swirl in the interior domain, while significant positive swirl exists in the region close to the cylinder wall. The vertical velocity shows the peaked structure close to the cylinder wall, indicating the singular eruption of the Ekman flux into the $E k^{1 / 3}$ layer, with accompanying negative velocity directly adjacent to close the recirculation. Furthermore, the radial inflow in the sidewall region can be observed here, corresponding to positive $u_{\theta}$. In this normalised plot this radial-velocity structure may be recognised, yet in absolute sense the radial velocity component is much smaller than the vertical and azimuthal contributions.

The description of the secondary circulation is only qualitative, as a full quantitative treatment would lead too far for the purpose of the current paper. A quantitative treatment of the secondary circulation will be presented in a forthcoming paper.

Additional evidence that the mean velocity profiles close to the sidewall are indeed governed by Stewartson boundary layer dynamics is provided in figure 11, where it is shown that the thickness of the sidewall boundary layers observed in the simulations follows the theoretical predictions (Kunnen et al. 2010a).

The role of Ekman and Stewartson boundary layer dynamics on the average mean flow is here illustrated with numerical simulations with $R a=1 \times 10^{9}$ and $\operatorname{Pr}=6.4$. Separate sets of simulations with $R a=2.73 \times 10^{8}$ with $\operatorname{Pr}=6.26$ and $R a=1 \times 10^{8}$ with $\operatorname{Pr}=6.4$ essentially revealed the same picture. The explanation provided in this section is therefore applicable to a range of Rayleigh numbers. Further studies are needed to explore the regime with $R a \gtrsim 1 \times 10^{9}$ and the role of the Prandtl number. We have also explored the role of the Rossby number, in particular by increasing the value of $1 / R o$ such that the flow is dominated by strong rotation (close to and even in regime III). The vertical vortex plumes then become stronger and data from azimuthally averaged components of velocity reveal an increasing number of alternating, vertically almost homogeneous regions of $u_{\phi}<0$ and $u_{\phi}>0$. 


\subsection{Influence of the secondary circulation on sidewall temperature measurements}

The above observations of the flow structure enable us to explain the sidewall temperature measurements. In figure 12 we have plotted radial distributions of the azimuthally averaged temperature and vertical velocity at $z=0.25 \mathrm{~L}$. Three main observations from the data extracted at this particular level are: a strong vertical mean flow near the sidewall (and for the highest rotation rate clearly within the $E k^{1 / 3}$ layer), an increase of the mean temperature in the bulk with the rotation rate, and an increased mean temperature near the sidewall compared to the bulk, regardless of the existence of the LSC. We start with the latter observation. In regime I (weak rotation) where the LSC is the dominant feature of the flow, the temperature gradient at the sidewall is caused by the LSC, which carries warm fluid upwards along the sidewall and cold fluid down in the middle (when considered in an azimuthally-averaged plot); and vice versa in the top half of the cylindrical domain. In regime II (moderate rotation) the LSC has disappeared and vertical vortices form the dominant feature of the flow. In this regime the secondary circulation described above causes a flow directed vertically away from the plate close to the sidewall, i.e. the singular eruption into the $E k^{1 / 3}$ layer, see figure 12 (b). This means that in regime II the secondary circulation carries warm fluid upwards along the sidewall in the lower half of the cylinder and cold fluid downwards along the sidewall in the top half. The vertical temperature gradient on the sidewall is increased. The enhanced mean temperature in the bulk with increasing rotation rate indicates that the vertical temperature gradient in the bulk increases also with increasing $1 / R o$, see for example figure 15 of Kunnen et al. (2010a). The bulk temperature gradient is caused by the merger of vertical plumes (see, e.g., Julien et al. 1996b, 1999; Legg et al. 2001; Sprague et al. 2006; Boubnov \& Golitsyn 1986: Zhong et al.|1993; Ecke \& Liu 1998: Sprague et al. 2006), i.e. the enhanced horizontal mixing of the temperature anomaly of the plumes results in a mean temperature gradient. In regime III this temperature gradient in the bulk becomes stronger than in regime II and eventually becomes equal to the temperature gradient at the sidewall.

\section{Conclusions}

Based on the experimental data and results obtained from DNS we studied the characteristics of the azimuthal temperature profiles at the sidewall. We find that in regime I (weak rotation) the LSC is the dominant feature of the flow. In the sidewall temperature measurements this is identified by the strong presence of the first Fourier mode in the signal. When the rotation is increased a sudden onset in the heat transport enhancement is found that indicates the beginning of regime II, the moderate rotation regime. In this regime the LSC is replaced by vertically aligned vortices and due to their random position the magnitude of the first Fourier mode is very weak, which confirms that the LSC has disappeared. This feature is also observed in regime III, where the heat transfer decreases, because the vertical velocity fluctuations are suppressed by the rotation.

When the LSC is the dominant feature of the flow the vertical temperature gradient at the sidewall is mainly due to plumes that travel close to the sidewall because they travel with the LSC. However, also in regime II and III, in which the LSC is absent, there is a strong temperature gradient at the sidewall. In these regimes, where the vertical vortices are the dominant feature of the flow, the observed vertical temperature gradient along the sidewall is mainly due to the secondary flow. The secondary flow, driven by the Ekman boundary layers near the plates, causes a recirculation in the Stewartson 


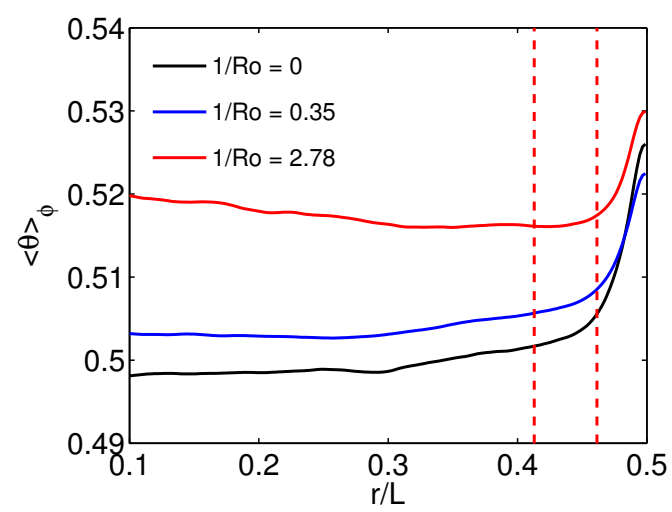

(a)

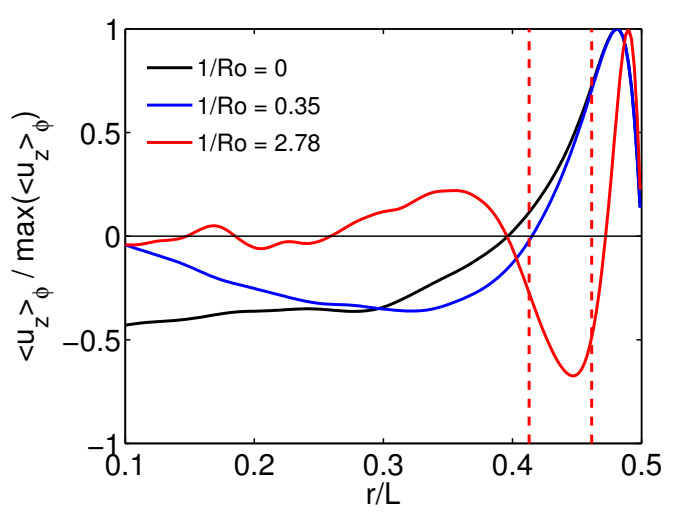

(b)

Figure 12. (a) Azimutally averaged temperature and (b) vertical velocity profiles as function of the radial position at the height $z=0.25 L$ for $R a=1.00 \times 10^{9}$ and $\operatorname{Pr}=6.4$. Black, blue, and red indicate the data for $1 / R o=0,1 / R o=0.35$, and $1 / R o=2.78$, respectively. The dashed lines indicate typical Stewartson boundary layer thicknesses $\left(\delta_{S, 1 / 3}=E k^{1 / 3}\right.$ and $\delta_{S, 1 / 4}=E k^{1 / 4}$ near the sidewall for $1 / R o=2.78\left(\delta_{S, 1 / 3}\right.$ is closest to the sidewall $)$. Note that for $1 / R o=2.78$ there is a strong upward flow of fluid in the inner Stewartson $E k^{1 / 3}$ layer, which causes a vertical temperature gradient at the sidewall. In panel (a) the increasing mean temperature in the bulk with increasing rotation rate indicates that the vertical temperature gradient in the bulk increases with increasing $1 / R o$. The temperature gradient in the bulk for this case is shown in figure 15 of Kunnen et al. $(2010 a)$.

boundary layer on the sidewall with upward (downward) transport of hot (cold) fluid close to the sidewall in the bottom (top) part of the cell.

It is remarkable that the secondary flow in turbulent RB convection, which is observed after time-averaging of the flow field, is so well described by linear Ekman and Stewartson boundary layer theory. It seems that the mean laminar flow profiles prevail, hidden under highly turbulent fluctuations. Thus the knowledge about laminar flow dynamics can still be highly relevant in the study of turbulent flows. An example is the Grossmann-Lohse (GL) theory (see Ahlers et al. 2009, for an overview), in which the kinetic energy and thermal variance dissipation rates have been decomposed into boundary-layer and bulk contributions. Scaling-wise and in a time-averaged sense a laminar Prandtl-Blasius profile is assumed close to the horizontal plates. The GL theory successfully describes the $N u$ and Re number dependences on Ra and Pr. Recently, Zhou \& Xia (2010) and Zhou et al. (2010) have shown that the laminar PB profile is indeed hidden under the turbulent fluctuations.

\section{Acknowledgements}

We gratefully acknowledge various discussions with Guenter Ahlers and Detlef Lohse over this line of research and their helpful comments on our manuscript. The authors wish to thank Eric de Cocq, Gerald Oerlemans, and Freek van Uittert (design and manufacturing of the experimental set-up) for their contributions to this project. Jaap van Wensveen of Tempcontrol is thanked for advice and for his help with the calibration of the thermistors. We thank Roberto Verzicco for providing the numerical code. R.J.A.M.S and R.P.J.K. wish to thank the Foundation for Fundamental Research on Matter (Stichting voor Fundamenteel Onderzoek der Materie, FOM) for financial support. This work was sponsored by the National Computing Facilities Foundation (NCF) for the use of super- 
computer facilities (Huygens), with financial support from the Netherlands Organization for Scientific Research (NWO).

\section{REFERENCES}

Ahlers, G., Grossmann, S. \& Lohse, D. 2009 Heat transfer and large scale dynamics in turbulent Rayleigh-Bénard convection. Rev. Mod. Phys. 81, 503.

van Bokhoven, L. J. A. 2007 Experiments on rapidly rotating turbulent flows. PhD thesis, Eindhoven University of Technology.

Boubnov, B. M. \& Golitsyn, G. S. 1986 Experimental study of convective structures in rotating fluids. J. Fluid Mech. 167, 503-531.

Boubnov, B. M. \& Golitsyn, G. S. 1990 Temperature and velocity field regimes of convective motions in a rotating plane fluid layer. J. Fluid Mech. 219, 215-239.

Brown, E. \& Ahlers, G. 2006 a Effect of the Earth's Coriolis force on turbulent RayleighBénard convection in the laboratory. Phys. Fluids 18, 125108-1 - 15.

Brown, E. \& Ahlers, G. $2006 b$ Rotations and cessations of the large-scale circulation in turbulent Rayleigh-Bénard convection. J. Fluid Mech. 568, 351-386.

Brown, E. \& Ahlers, G. 2007 Large-scale circulation model for turbulent Rayleigh-Bénard convection. Phys. Rev. Lett. 98, 134501.

Brown, E., Funfschilling, D., Nikolaenko, A. \& Ahlers, G. 2005 a Heat transport by turbulent Rayleigh-Bénard convection: Effect of finite top- and bottom conductivity. Phys. Fluids 17, 075108.

Brown, E., Nikolaenko, A. \& Ahlers, G. $2005 b$ Reorientation of the large-scale circulation in turbulent Rayleigh-Bénard convection. Phys. Rev. Lett. 95, 084503.

Ecke, R. E. \& Liu, Y. 1998 Traveling-wave and vortex states in rotating Rayleigh-Bénard convection. Int. J. Eng. Sci. 36, 1471-1480.

Fernando, H. J. S., Chen, R. \& Boyer, D. L. 1991 Effects of rotation on convective turbulence. J. Fluid Mech. 228, 513-547.

Funfschilling, D., Brown, E., Nikolaenko, A. \& Ahlers, G. 2005 Heat transport by turbulent Rayleigh-Bénard convection in cylindrical cells with aspect ratio one and larger. J. Fluid Mech. 536, 145-154.

Greenspan, H. P \& Howard, L. N. 1963 On a time-dependent motion of a rotating fluid. $J$. Fluid Mech. 17, 385-404.

Hart, J. E., Kittelman, S. \& Ohlsen, D. R. 2002 Mean flow precession and temperature probability density functions in turbulent rotating convection. Phys. Fluids 14, 955-962.

HART, J. E. \& Olsen, D. R. 1999 On the thermal offset in turbulent rotating convection. Phys. Fluids 11, 2101-2107.

van HeIJst, G. J. F. 1983 The shear-layer structure in a rotating fluid near a differentially rotating sidewall. J. Fluid Mech. 130, $1-12$.

VAn Heisst, G. J. F. 1984 Source-sink flow in a rotating cylinder. J. Engng Math. 18, 247 257.

VAn HeiJst, G. J. F. 1986 Fluid flow in a partially-filled rotating cylinder. J. Engng Math. 20, $233-250$.

Homsy, G. M. \& Hudson, J. L. 1969 Centrifugally driven thermal convection in a rotating cylinder. J. Fluid Mech. 35, 33-52.

Julien, K., LegG, S., McWilliams, J. \& Werne, J. $1996 a$ Hard turbulence in rotating Rayleigh-Bénard convection. Phys. Rev. E 53, R5557-R5560.

Julien, K., LegG, S., McWilliams, J. \& Werne, J. 1996b Rapidly rotating Rayleigh-Bénard convection. J. Fluid Mech. 322, 243-273.

Julien, K., LegG, S., McWilliams, J. \& Werne, J. 1999 Plumes in rotating convection. part 1. ensemble statistics and dynamical balances. J. Fluid Mech. 391, 151-187.

King, E. M., Stellmach, S., Noir, J., Hansen, U. \& Aurnou, J. M. 2009 Boundary layer control of rotating convection systems. Nature 457, 301.

Kunnen, R.P.J., Geurts, B.J. \& Clercx, H.J.H. 2009 Turbulence statistics and energy budget in rotating Rayleigh-Bénard convection. Eur. J. Mech. B/Fluids 28, 578 - 589. 
Kunnen, R. P. J., Clercx, H. J. H. \& Geurts, B. J. 2006 Heat flux intensification by vortical flow localization in rotating convection. Phys. Rev. E 74, 056306.

Kunnen, R. P. J., Clercx, H. J. H. \& Geurts, B. J. 2008 a Breakdown of large-scale circulation in turbulent rotating convection. Europhys. Lett. 84, 24001.

Kunnen, R. P. J., Clercx, H. J. H. \& Geurts, B. J. $2008 b$ Enhanced vertical inhomogeneity in turbulent rotating convection. Phys. Rev. Lett. 101, 174501.

Kunnen, R. P. J., Geurts, B. J. \& Clercx, H. J. H. 2010 a Experimental and numerical investigation of turbulent convection in a rotating cylinder. J. Fluid Mech. 642, 445-476.

Kunnen, R. P. J., Geurts, B. J. \& Clercx, H. J. H. $2010 b$ Vortex statistics in turbulent rotating convection. Phys. Rev. E 82, 036306.

LegG, S., Julien, K., McWilliams, J. \& Werne, J. 2001 Vertical transport by convection plumes: modification by rotation. Phys. Chem. Earth (B) 26, 259-262.

LiU, Y. \& Ecke, R. E. 1997 Heat transport scaling in turbulent Rayleigh-Bénard convection: effects of rotation and Prandtl number. Phys. Rev. Lett. 79, 2257-2260.

LiU, Y. \& ECKE, R. E. 2009 Heat transport measurements in turbulent rotating RayleighBénard convection. Phys. Rev. E 80, 036314.

Lohse, D. \& XIA, K. Q. 2010 Small-scale properties of turbulent Rayleigh-Bénard convection. Annu. Rev. Fluid Mech. 42, 335-364.

Moore, D. W. \& Saffman, P. G. 1969 The shear-layer structure in a rotating fluid near a differentially rotating sidewall. Phil. Trans. R. Soc. A 264, 597-634.

Niemela, J.J., Babuin, S. \& Sreenivasan, K.R. 2010 Turbulent rotating convection at high Rayleigh and Taylor numbers. J. Fluid Mech. 649, 509.

QIU, X. L. \& Tong, P. 2001 Large scale velocity structures in turbulent thermal convection. Phys. Rev. E 64, 036304.

Rossby, H. T. 1969 A study of Bénard convection with and without rotation. J. Fluid Mech. 36, 309-335.

SAKAI, S. 1997 The horizontal scale of rotating convection in the geostrophic regime. J. Fluid Mech. 333, 85-95.

Schmitz, S. \& Tilgner, A. 2009 Heat transport in rotating convection without Ekman layers. Phys. Rev. E 80, 015305.

Schmitz, S. \& Tilgner, A. 2010 Transitions in turbulent rotating Rayleigh-Bénard convection. Geophys. Astrophys. Fluid Dyn. 104, 1029-0419.

Sprague, M., Julien, K., Knobloch, E. \& Werne, J. 2006 Numerical simulation of an asymptotically reduced system for rotationally constrained convection. J. Fluid Mech. 551, $141-174$.

Stevens, R. J. A. M., Clercx, H. J. H. \& Lohse, D. $2010 a$ Boundary layers in rotating weakly turbulent Rayleigh-Bénard convection. Phys. Fluids 22, 085103.

Stevens, R. J. A. M., Clercx, H. J. H. \& Lohse, D. $2010 b$ Effect of plumes on measuring the large-scale circulation in turbulent Rayleigh-Bénard convection. Phys. Fluids, (in press).

Stevens, R. J. A. M., Clercx, H. J. H. \& Lohse, D. $2010 c$ Optimal Prandtl number for heat transfer in rotating Rayleigh-Bénard convection. New J. Phys. 12, 075005.

Stevens, R. J. A. M., Zhong, J.-Q., Clercx, H. J. H., Ahlers, G. \& Lohse, D. 2009 Transitions between turbulent states in rotating Rayleigh-Bénard convection. Phys. Rev. Lett. 103, 024503.

Stewartson, K. 1957 On almost rigid rotations. J. Fluid Mech. 3, 17-26.

Stewartson, K. 1966 On almost rigid rotations. Part 2. J. Fluid Mech. 26, 131-144.

Verzicco, R. \& CAmussi, R. 1997 Transitional regimes of low-prandt thermal convection in a cylindrical cell. Phys. Fluids 9, 1287-1295.

VerzicCo, R. \& CAmussi, R. 2003 Numerical experiments on strongly turbulent thermal convection in a slender cylindrical cell. J. Fluid Mech. 477, 19-49.

Verzicco, R. \& Orlandi, P. 1996 A finite-difference scheme for three-dimensional incompressible flow in cylindrical coordinates. J. Comput. Phys. 123, 402-413.

Vorobieff, P. \& Ecke, R. E. 2002 Turbulent rotating convection: an experimental study. $J$. Fluid Mech. 458, 191-218.

Weiss, S., Stevens, R.J.A.M., Zhong, J.-Q., Clercx, H.J.H., Lohse, D. \& Ahlers, G. 2010 Finite-size effects lead to supercritical bifurcations in turbulent rotating RayleighBénard convection. Phys. Rev. Lett. 105, 224501. 
Zhong, F., Ecke, R. E. \& Steinberg, V. 1993 Rotating Rayleigh-Bénard convection: asymmetrix modes and vortex states. J. Fluid Mech. 249, 135-159.

Zhong, J.-Q. \& Ahlers, G. 2010 Heat transport and the large-scale circulation in rotating turbulent Rayleigh-Bénard convection. J. Fluid Mech. 665, 300-333.

Zhong, J.-Q., Stevens, R. J. A. M., Clercx, H. J. H., Verzicco, R., Lohse, D. \& Ahlers, G. 2009 Prandtl-, Rayleigh-, and Rossby-number dependence of heat transport in turbulent rotating Rayleigh-Bénard convection. Phys. Rev. Lett. 102, 044502.

Zhou, Q., Stevens, R. J. A. M., Sugiyama, K., Grossmann, S., Lohse, D. \& Xia, K.Q. 2010 Prandtl-Blasius temperature and velocity boundary layer profiles in turbulent Rayleigh-Bénard convection. J. Fluid Mech. 664, 297-312.

Zhоu, Q. \& XIA, K.-Q. 2010 Measured instantaneous viscous boundary layer in turbulent Rayleigh-Bénard convection. Phys. Rev. Lett. 104, 104301. 